\title{
SR AND ND ISOTOPIC DIFFERENCE BETWEEN KIMBERLITES AND CARBONATITES FROM SIBERIA
}

\author{
TOSHIRo MORIKIYO ${ }^{1}$, SERGEY I.KOSTROVITSKY ${ }^{2}$, MORAGalleW.K.WEERAKOON ${ }^{3}$,

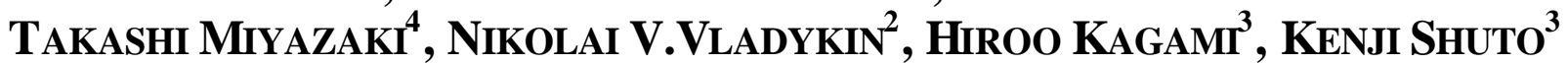 \\ ${ }^{1}$ Shinshu University, Japan; ${ }^{2}$ Institute of geochemistry of Siberian Branch, Irkutsk, Russia; ${ }^{3}$ Niigata University, Japan; \\ ${ }^{4}$ Institute for Geothermal Sciences, Kyoto University, Japan
}

Whole-rock $\mathrm{Sr}$ and $\mathrm{Nd}$ isotopic compositions of 31 kimberlite samples and 38 carbonatite samples from the Siberian Platform were obtained. Initial $\mathrm{Sr}$ and $\mathrm{Nd}$ isotopic ratios were calculated by using ages from literatures.

The aims of the study are

to elucidate the general features in $\mathrm{Sr}$ and $\mathrm{Nd}$ isotopic ratios for kimberlites occurring in the Yakutia district,

to compare $\mathrm{Sr}$ and $\mathrm{Nd}$ isotopic features of kimberlites with those of carbonatites in order to examine the genetic relationship between the rocks.

The kimberlites in the Yakutia province occur in the four different zones; Zone 1 (Olenyok-Anabar zone), Zone 2 (Daldyn-Olenyok zone), Zone 3 (ViluyMarhinsk zone), and Zone 4 (South boundary of the Siberian platform). The distribution of those zones and the location of each kimberlite field are described in Afanasiev et al., 1995. The estimated ages of the kimberlites are shown in Table 1. Kimberlite samples investigated in this study were taken from all the zones above.

Carbonatites occurring in the Siberian Platform have been classified into two groups on the basis of their initial $\mathrm{Sr}$ and $\mathrm{Nd}$ isotopic signatures; Group 1 carbonatites have isotopically depleted feature whereas Group 2 carbonatites have enriched feature (Morikiyo et al., 2001). The Guli, Essei, Kiiskii, Srednetatarskii, Bolshetagninskii, Nizhnesayanskii, Vehnesayanskii, Zhidoy, Arbarastah, Ingili, Ozernyi and Koksharovskii carbonatite bodies are classified as Group 1. The Malomurunnskii and Khani bodies are Group 2. It should be noted that the age of carbonatite does not correlate with that of kimberlite, and that an occurrence of carbonatite is not found in the kimberlite fields above, with the exception of Anabar province (Kogarko et al., 1995).

The isotope analytical results are as follows: Kimberlites have initial eNd values ranging from +1.6 to +4.6 , with the exception of the sample from Ingashi field. The initial $\mathrm{eSr}$ values range from -14.8 to +44.4 (Table 2). Data points are scattered within the upper quadrant of the eNd vs. eSr diagram. But a linear arrangement parallel to the horizontal axis (eSr axis) is seen. For example, the eNd values for the samples from the Kuoika kimberlite field (Zone 2) are nearly constant $(+3.5)$, but their eSr are variable, ranging from -11 to
+28 (Fig.1). This radiogenic Sr enrichment is interpreted as being due to the results of isotopic exchange with ground waters having high ${ }^{87} \mathrm{Sr} /{ }^{86} \mathrm{Sr}$ ratios. Thus we exclude such data from our consideration. Then it is seen that the unaltered kimberlites from the Siberian Platform have isotopic compositions ranging from -1.6 to +4.6 for initial eNd values, and from -14.8 to +2.4 for initial e Sr values (Fig.2). This compositional range is essentially similar to that of Group 1 kimberlite in South Africa.

The isotopic data for Group I carbonatites fall in a narrow field in the depleted quadrant in the eNd vs. eSr diagram. They have initial eNd values ranging from +2.7 to +7.0 , and initial eSr ranging from -20 to 4(Morikiyo et al., 2001; Fig.2). One-half of the carbonatite samples of Group 1 have eNd values higher than +4.6. By contrast, all the kimberlites have eNd values lower than +4.6. Thus, it is clear that kimberlites and carbonatites were not derived from the same source material. It is concluded that kimberlite and carbonatite are not genetically related.

Table 1: List of kimberlite fields of the Siberian Platform

\begin{tabular}{|l|l|l|l|}
\hline Zone & & $\begin{array}{l}\text { Kimberlite } \\
\text { field }\end{array}$ & Age(Ma) \\
\hline 1 & $\begin{array}{l}\text { Olenyok-Anabar } \\
\text { zone }\end{array}$ & $\begin{array}{l}\text { Ari- } \\
\text { Mastah, } \\
\text { Luchakan, } \\
\text { D'uken }\end{array}$ & $\begin{array}{l}\text { Mesozoic } \\
(220,180 ?)\end{array}$ \\
\hline 2 & $\begin{array}{l}\text { Daldyn-Olenyok } \\
\text { zone }\end{array}$ & $\begin{array}{l}\text { Daldin, } \\
\text { Alakit }\end{array}$ & $\begin{array}{l}\text { Paleozoic } \\
(360)\end{array}$ \\
\hline 2 & $\begin{array}{l}\text { ditto } \\
\text { Kuoika }\end{array}$ & $\begin{array}{l}\text { Mesozoic } \\
(110-140)\end{array}$ \\
\hline 3 & $\begin{array}{l}\text { Viluy-Marhinsk } \\
\text { zone }\end{array}$ & $\begin{array}{l}\text { Malo- } \\
\text { Botuobin }\end{array}$ & $\begin{array}{l}\text { Paleozoic } \\
(360)\end{array}$ \\
\hline $\begin{array}{l}\text { South boundary } \\
\text { Ingashi } \\
\text { platform }\end{array}$ & $\begin{array}{l}\text { Proterozoic } \\
(1268)\end{array}$ \\
\hline & \multicolumn{2}{|l|}{} \\
\hline
\end{tabular}


Table 2a: Nd isotopic results for kimberlites

\begin{tabular}{|c|c|c|c|c|c|c|}
\hline Sample & $\begin{array}{l}\text { Sm } \\
\text { (ppm }\end{array}$ & $\begin{array}{l}\text { (ppm } \\
\text { ) }\end{array}$ & $\begin{array}{l}{ }^{147} \mathrm{Sm} / \\
{ }^{144} \mathrm{Nd} \\
\text { (atom- } \\
\text { ic) }\end{array}$ & $\begin{array}{l}{ }^{143} \mathrm{Nd} / \\
{ }^{144} \mathrm{Nd} \\
\text { (meas- } \\
\text { ured) }\end{array}$ & $\begin{array}{l}{ }^{143} \mathrm{Nd} / \\
{ }^{144} \mathrm{Nd} \\
\text { (initial) }\end{array}$ & åNd \\
\hline \multicolumn{7}{|c|}{ Ari-Mastah(220Ma) } \\
\hline 78-1534 & 11.4 & 77.2 & 0.0891 & 0.51259 & 0.51246 & 2.1 \\
\hline \multicolumn{7}{|c|}{ Malo-Kuonamk (220Ma) } \\
\hline 78-1003 & 19.4 & 146 & 0.0807 & 0.51267 & 0.51255 & 3.8 \\
\hline 78-1185 & 18.1 & 135 & 0.0809 & 0.51264 & 0.51252 & 3.2 \\
\hline 78-1215 & 9.01 & 65.7 & 0.0830 & 0.51259 & 0.51247 & 2.2 \\
\hline \multicolumn{7}{|c|}{ Luchakan (220Ma) } \\
\hline 78-1380 & 14.1 & 101 & 0.0849 & 0.51261 & 0.51249 & 2.6 \\
\hline 78-1395 & 25.2 & 111 & 0.1366 & 0.51259 & 0.51239 & 0.7 \\
\hline 99-3 & 20.7 & 148 & 0.0849 & 0.51240 & 0.51227 & -1.6 \\
\hline $99-14$ & 25.4 & 172 & 0.0892 & 0.51262 & 0.51249 & 2.6 \\
\hline \multicolumn{7}{|c|}{ Starorechinsk (180?Ma) } \\
\hline 78-1565 & 105 & 913 & 0.0695 & 0.51266 & 0.51258 & 3.4 \\
\hline $90-67$ & 10.2 & 82.7 & 0.0747 & 0.51269 & 0.51260 & 3.8 \\
\hline 78-1610 & 13.5 & 109 & 0.0746 & 0.51269 & 0.51260 & 3.8 \\
\hline \multicolumn{7}{|c|}{ Haramay (250Ma) } \\
\hline Xp-10 & 9.33 & 68.2 & 0.0827 & 0.51253 & 0.51240 & 1.6 \\
\hline Xp-8 & 16.4 & 120 & 0.0831 & 0.51255 & 0.51241 & 1.8 \\
\hline Xp-11 & 19.3 & 142 & 0.0822 & 0.51252 & 0.51239 & 1.4 \\
\hline \multicolumn{7}{|c|}{ Ingashi (1268Ma) } \\
\hline NH-1 & 29.8 & 286 & 0.0631 & 0.51100 & 0.51048 & -10.3 \\
\hline \multicolumn{7}{|c|}{ Daldin (360Ma) } \\
\hline 01-280 & 10.8 & 81.5 & 0.0802 & 0.51257 & 0.51238 & 4.0 \\
\hline \multicolumn{7}{|c|}{ Alakit (360Ma) } \\
\hline 01-361 & 11.8 & 114 & 0.0624 & 0.51254 & 0.51239 & 4.3 \\
\hline \multicolumn{7}{|c|}{ Malo-Botuobin (360Ma) } \\
\hline $00 / 289$ & 12.1 & 87.4 & 0.0835 & 0.51261 & 0.51241 & 4.6 \\
\hline \multicolumn{7}{|c|}{ Verhna-Muna (360Ma) } \\
\hline $9-200$ & 10.6 & 68.1 & 0.0943 & 0.51262 & 0.51240 & 4.3 \\
\hline $9-154$ & 12.8 & 92.8 & 0.0831 & 0.51247 & 0.51227 & 1.9 \\
\hline \multicolumn{7}{|c|}{ Kuoika (110-140Ma) } \\
\hline 7-191 & 20.9 & 125 & 0.1012 & 0.51272 & 0.51263 & 3.4 \\
\hline $7-483$ & 32.4 & 248 & 0.0790 & 0.51271 & 0.51264 & 3.5 \\
\hline $7-487$ & 9.52 & 65.6 & 0.0877 & 0.51278 & 0.51270 & 4.6 \\
\hline 7-78 & 9.47 & 67.8 & 0.0844 & 0.51274 & 0.51267 & 4.1 \\
\hline 7-384 & 15.7 & 117 & 0.0809 & 0.51271 & 0.51263 & 3.4 \\
\hline 7-388 & 4.06 & 30.0 & 0.0817 & 0.51272 & 0.51264 & 3.6 \\
\hline \multicolumn{7}{|c|}{ South Anabar (220Ma) } \\
\hline 001-11 & 15.0 & 93.8 & 0.0964 & 0.51247 & 0.51233 & -0.4 \\
\hline \multicolumn{7}{|c|}{ Chomurdah(360Ma) } \\
\hline $7-460$ & 15.9 & 97.9 & 0.0985 & 0.51256 & 0.51233 & 3.1 \\
\hline \multicolumn{7}{|c|}{ D'uken (220Ma) } \\
\hline 97-192 & 20.8 & 136 & 0.0923 & 0.51266 & 0.51253 & 3.4 \\
\hline 97-128 & 37.2 & 261 & 0.0862 & 0.51267 & 0.51255 & 3.7 \\
\hline 97-90 & 18.6 & 115 & 0.0981 & 0.51267 & 0.51253 & 3.4 \\
\hline
\end{tabular}

Table 2b: Sr isotopic results for kimberlites

\begin{tabular}{|c|c|c|c|c|c|c|}
\hline Sample & $\begin{array}{l}\mathbf{R b} \\
(\mathbf{p p m} \\
)\end{array}$ & $\begin{array}{l}\mathrm{Sr}_{86}^{87} \\
\text { (ppm } \\
)\end{array}$ & $\begin{array}{l}\mathrm{Rb} / \\
\mathrm{Sr} \\
\text { (atom- } \\
\text { ic) }\end{array}$ & $\begin{array}{l}{ }^{87} \mathbf{S r} / \\
{ }^{86} \mathrm{Sr} \\
\text { (meas- } \\
\text { ured) }\end{array}$ & $\begin{array}{l}{ }^{87} \mathrm{Sr} / \\
\text { (initial) }\end{array}$ & $\begin{array}{l}\text { å } \\
\mathbf{S} \mathbf{r}\end{array}$ \\
\hline Ari- & Gastah & $(220 \mathrm{M}$ & & & & \\
\hline $8-1534$ & 67.5 & 595 & 0.3281 & 0.70503 & $\mathbf{0 . 7 0 4 0 0}$ & -3.4 \\
\hline Malo- & Kuona & nk (22 & 0Ma) & & & \\
\hline 78-1003 & 55.3 & 1440 & 0.1109 & 0.70416 & $\mathbf{0 . 7 0 3 8 1}$ & -6.1 \\
\hline 8-1185 & 72.3 & 806 & 0.2595 & 0.70401 & $\mathbf{0 . 7 0 3 2 0}$ & -14.8 \\
\hline 78-1215 & 31.1 & 1170 & 0.0769 & 0.70626 & 0.70602 & 25.2 \\
\hline Lucha & $\operatorname{kan}(2$ & 20Ma) & & & & \\
\hline 78-1380 & 141 & 635 & 0.6442 & 0.70587 & $\mathbf{0 . 7 0 3 8 5}$ & -5.5 \\
\hline 78-1395 & 74.2 & 776 & 0.2765 & 0.70445 & 0.70359 & -9.3 \\
\hline 99-3 & 55.2 & 785 & 0.2033 & 0.70445 & 0.70381 & -6.1 \\
\hline $99-14$ & 39.7 & 1360 & 0.0847 & 0.70401 & 0.70375 & -7.0 \\
\hline
\end{tabular}

Starorechinsk (180?Ma)

$\begin{array}{llllllll}78-1565 & 25.6 & 2730 & 0.0271 & 0.70364 & 0.70358 & -10.1\end{array}$

$\begin{array}{llllllll}90-67 & 36.5 & 722 & 0.1462 & 0.70387 & 0.70349 & -11.3\end{array}$

$\begin{array}{llllllll}78-1610 & 33.8 & 741 & 0.1319 & 0.70459 & 0.70426 & -0.5\end{array}$

Haramay (250Ma)

$\begin{array}{lllllll}\text { Xp-10 } & 31.7 & 601 & 0.1525 & 0.70513 & 0.70459 & 5.4\end{array}$

$\begin{array}{llllllll}\text { Xp-8 } & 84.5 & 304 & 0.8042 & 0.70680 & 0.70394 & -3.8\end{array}$

$\begin{array}{llllllll}X p-11 & 74.7 & 1550 & 0.1392 & 0.70460 & 0.70411 & -1.4\end{array}$

Ingashi (1268Ma)

$\begin{array}{lllllll}\text { NH-1 } & 84.3 & 2580 & 0.0944 & 0.70783 & 0.70613 & 44.4\end{array}$

Daldin (360Ma)

$\begin{array}{lllllll}01-280 & 18.9 & 1110 & 0.0494 & 0.70460 & 0.70435 & 3.9\end{array}$

Alakit (360Ma)

$\begin{array}{lllllll}01-361 & 27.2 & 749 & 0.1052 & 0.70465 & 0.70411 & 0.5\end{array}$

Malo-Botuobin (360Ma)

$\begin{array}{lllllll}00 / 289 & 21.8 & 668 & 0.0945 & 0.70391 & 0.70342 & -9.3\end{array}$

Verhna-Muna (360Ma)

$\begin{array}{lllllll}9-200 & 117 & 1000 & 0.3362 & 0.70611 & 0.70439 & 4.4\end{array}$

$\begin{array}{llllllll}9-154 & 93.5 & 1810 & 0.1497 & 0.70717 & 0.70641 & 33.1\end{array}$

Kuoika (110-140Ma)

$\begin{array}{rrrrrrr}7-191 & 89.3 & 1120 & 0.2302 & 0.70441 & 0.70395 & -5.4\end{array}$

$\begin{array}{llllllll}7-483 & 15.2 & 849 & 0.0518 & 0.70430 & 0.70420 & -1.9\end{array}$

$\begin{array}{lllllllll}7-487 & 115 & 749 & 0.4436 & 0.70446 & 0.70358 & -10.8\end{array}$

$\begin{array}{llllllll}7-78 & 43.3 & 1220 & 0.1027 & 0.70464 & 0.70444 & 1.5\end{array}$

$\begin{array}{rrrrrrrr}7-384 & 72.6 & 2190 & 0.0958 & 0.70527 & 0.70508 & 10.6\end{array}$

$\begin{array}{llllllll}7-388 & 155 & 511 & 0.8761 & 0.70806 & 0.70632 & 28.1\end{array}$

South Anabar (220Ma)

$\begin{array}{lllllll}001-11 & 135 & 2060 & 0.1898 & 0.70500 & 0.70441 & 2.4\end{array}$

Chomurdah(360Ma)

$\begin{array}{lllllll}7-460 & 66.8 & 1010 & 0.1920 & 0.70601 & 0.70502 & 13.4\end{array}$

D'uken (220Ma)

$\begin{array}{llllllll}97-192 & 44.2 & 825 & 0.1548 & 0.70430 & 0.70382 & -6.1\end{array}$

$\begin{array}{llllllll}97-128 & 88.2 & 649 & 0.3932 & 0.70506 & 0.70383 & -5.9\end{array}$

\begin{tabular}{llllllll}
$97-90$ & 81.8 & 1110 & 0.2133 & 0.70449 & 0.70382 & -5.9 \\
\hline
\end{tabular} 


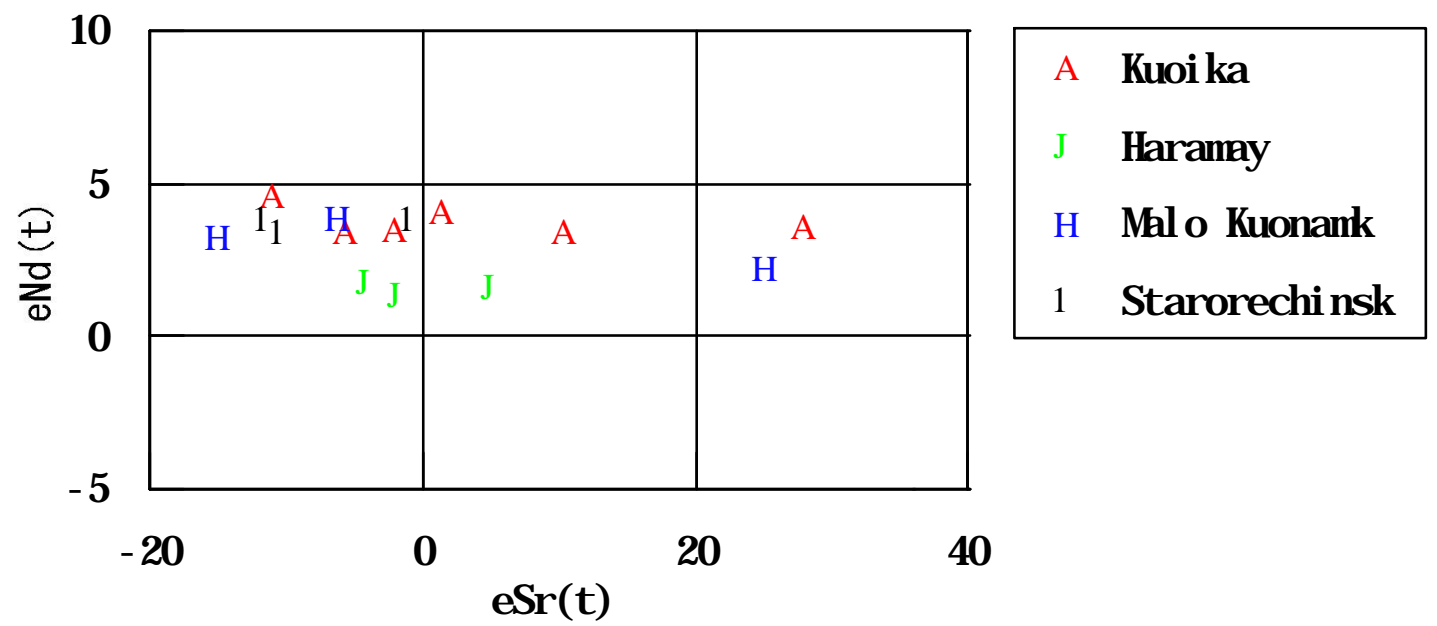

Figure 1: Initial eNd versus eSr values for kimberlites from Kuoika (square), Haramay (circle), Malo Kuonamk (triangle), and Starorechinsk (cross) kimberlite fields. Data from each kimberlite field plot parallel to abscissa with nearly the same eNd value. This indicates that $\mathrm{Sr}$ isotopic compositions of some kimberlite bodies were increased due to the reaction with groundwaters after the emplacement of kimberlites.

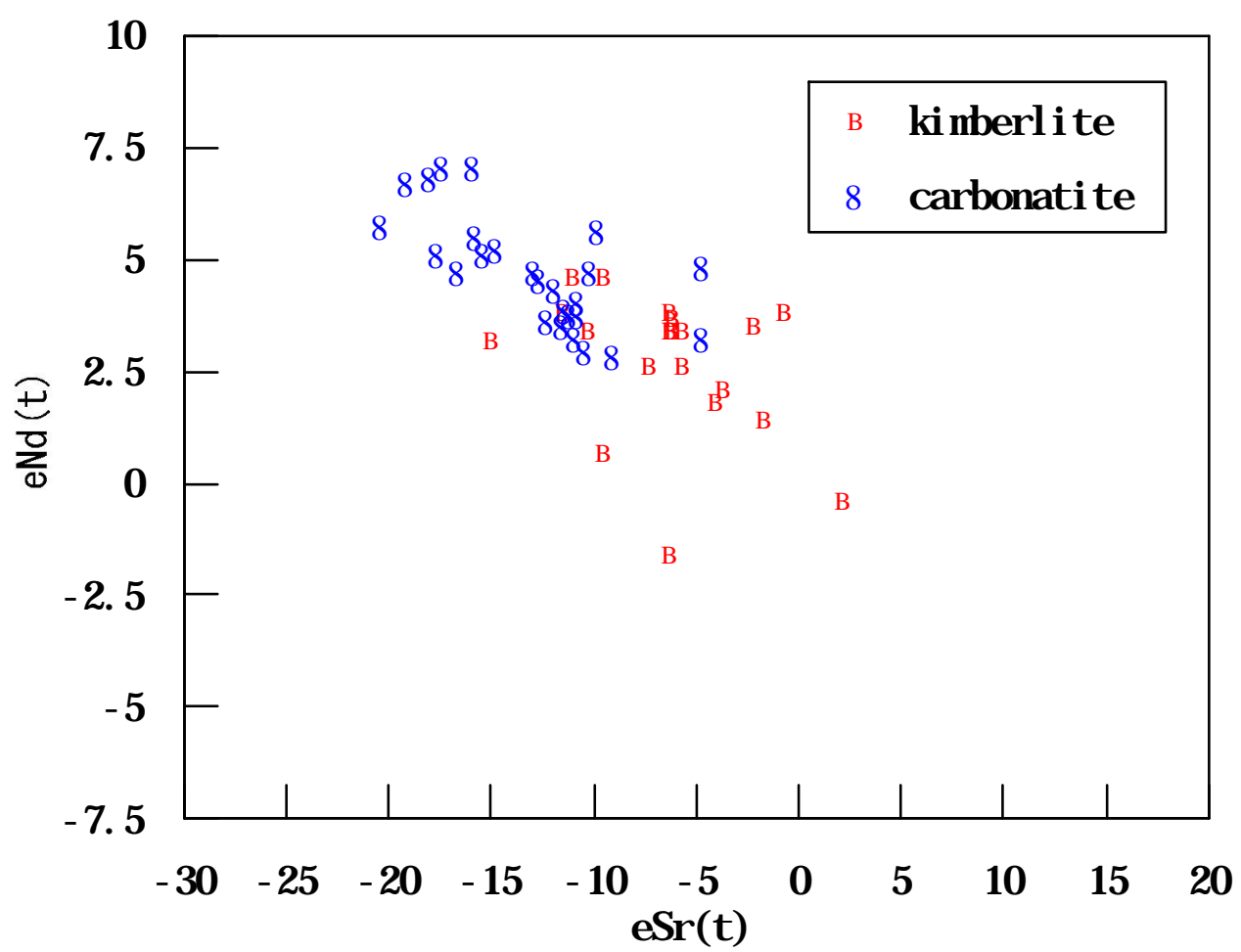

Figure 2: Initial eNd versus initial eSr values for kimberlites (square) and carbonatites (cross) from the Siberian Platform. Kimberlite samples probably underwent isotopic alteration have been excluded from the consideration. Carbonatites shown in this figure are those of Group 1 carbonatites of Morikiyo et al., (2001).
All of the kimberlite samples have eNd values lower than +4.6, whereas half of the carbonatite samples $(n=14)$ have eNd values higher than +4.6 . Carbonatites have more depleted $\mathrm{Nd}$ and $\mathrm{Sr}$ isotopic signatures than kimberlites. 
Table 3: Parameters used in the calculation of epsilon values

\begin{tabular}{|c|c|}
\hline $\mathbf{S r}$ & Nd \\
\hline $\begin{array}{l}\left({ }^{87} \mathrm{Sr} /{ }^{86} \mathrm{Sr}\right) \mathrm{pr}=\mathbf{0 . 7 0 4 5} \\
\left({ }^{87} \mathrm{Rb} /{ }^{86} \mathrm{Sr}\right) \mathrm{pr}=\mathbf{0 . 0 8 2 7}\end{array}$ & $\begin{array}{l}\left({ }^{143} \mathrm{Nd} /{ }^{144} \mathrm{Nd}\right) \mathrm{pr}=\mathbf{0 . 5 1 2 6 3 8} \\
\left({ }^{147} \mathrm{Sm} /{ }^{144} \mathrm{Nd}\right) \mathrm{pr}=\mathbf{0 . 1 9 6 6}\end{array}$ \\
\hline$\ddot{e}^{87} R b=1.42 \times 10^{-11} / y$ & $\ddot{e}^{147} \mathrm{Sm}=6.54 \times 10^{-12} / \mathrm{y}$ \\
\hline
\end{tabular}

\section{REFERENCES}

Afanasiev, V.P., Kryuchkov, A.I., Cherny, S.D., 1995. Geographic position and geological characteristics of the field trip area. In: Sobolev, N.V., Zuev, V.M., Pokhilenko, N.P., Zinchuk, N.N. (Eds.), Field Guide Book Kimberlites of Yakutia. Novosibirsk, pp.9-11.

Agashev, A.M., Orihashi, Y., Watanabe, T., Pokhilenko, N.P., Serenko, V.P., 2000. Isotopegeochemical features of the Siberian platform kimberlites in connection with the problem of their origin. Russian Geology and Geophysics, 41, 87-97.

Kogarko, L.N., Kononova, V.A., Orlova, M.P., Wooley, A.R., 1995. Alkaline rocks and carbonatites of the world Part 2: Former USSR. Chapman and Hall, London.

Kostrovitsky, S.I., Morikiyo, T., Vladykin, N.V., Lepin, V.S., 1999. Sr-Nd isotopic systematics on kimberlites and related rocks from the Yakutsk province, Siberia.. Dokl. Akad. Nauk SSSR 369, 371-374 (in Russian).

Kramers, J.D, Smith, C.B., Lock, N.P., Harmon, R.S., Boyd, F.R. 1981, Can Kimberlite be generated from an ordinary mantle? Nature, 291, 53-56.

Morikiyo,T.., Miyazaki, T., Kagami, H., Vladykin, N.V., Chernysheva, E.A., Panina, L.I., Podgornych, N.M., 2001. Sr, Nd, C and O isotope characteristics of Siberian carbonatites. In: Vladykin, N.V. (Ed.), Alkaline magmatism and problems of mantle sources, Irkutsk, pp. 6984.

Smith, C.B., 1983. Pb, Sr and $\mathrm{Nd}$ isotopic evidence for sources of southern African Cretaceous kimberlites. Nature 304, 51-54.

Contact: T Morikiyo, Shinshu University, Matsumoto, 390-8621, Japan, E-mail: xmoriki@gipac.shinshuu.ac.jp 